\title{
Clinical predictors of a positive test result in patients undergoing genetic evaluation for a hereditary kidney cancer syndrome
}

Andrea Kokorovic ${ }^{1}$; Aidan Thomas ${ }^{2}$; Jesus Serrano-Lomelin ${ }^{3}$; Meghan Ferguson ${ }^{2,4}$; Ricardo A Rendon $^{1}$

${ }^{1}$ Department of Urology, Dalhousie University, Halifax, NS, Canada; ${ }^{2}$ Maritime Medical Genetics Service, IWK Health Centre, Halifax, NS, Canada; ${ }^{3}$ Department of Obstetrics and Gynecology, University of Alberta, Edmonton, AB, Canada; ${ }^{4}$ MyGeneTeam, LLC, Miami, FL, United States

Cite as: Can Urol Assoc J 2020 March 23; Epub ahead of print. http://dx.doi.org/10.5489/cuaj.6264

Published online March 23, 2020

$* * *$

\section{Abstract}

Introduction: Guidelines are available to assist providers in identifying patients with renal cell carcinoma (RCC) that may benefit from genetic counselling, however, the evidence for these recommendations lacks support from the literature and controversy remains as to who should be referred. We aimed to delineate risk factors associated with a positive genetic test in a real-life cohort of patients with RCC referred to a regional medical genetics unit for evaluation of a hereditary kidney cancer syndrome.

Methods: Patients with a diagnosis of RCC referred to Maritime Medical Genetics Service (Nova Scotia, Canada) from 2006-2017 were reviewed using retrospective data. The primary outcome was identification of clinical features that were associated with a positive test result. Logistic regression models were used for analysis.

Results: A total of 135 patients were referred to medical genetics for evaluation; 102 patients were evaluated, 75 underwent testing, and 74 were included in the final analysis. Five patients tested positive: three Birt Hogg Dube, one Cowden syndrome, and one Von Hippel Lindau. Presence of dermatological lesions (specifically fibrofolliculomas) and more than two high-risk features were the only predictors of a positive test result.

Conclusions: The presence of dermatological lesions and more than two high-risk features are the only predictors of a positive test result in patients with a suspected hereditary kidney cancer syndrome. These findings are not reflected in current guidelines, and the clinical implementation of our results may improve the identification of high-risk patients for genetic counselling. 


\section{Introduction}

An estimated 7200 new cases of kidney cancer are diagnosed in Canada each year, with the majority representing renal cell carcinoma $(\mathrm{RCC})^{1}$. RCC entails various histological subtypes, including clear cell ( $75 \%$ of cases), papillary, chromophobe, collecting duct and unclassified ${ }^{2}$. RCC has been linked with several hereditary cancer syndromes, which harbour known germline mutations and carry distinct clinical manifestations ${ }^{3}$. They include von Hippel-Lindau (VHL), hereditary leiomyomatosis and renal cell carcinoma (HLRCC), hereditary papillary renal cell carcinoma (HPRCC), Birt-Hogg-Dube syndrome (BHD), tuberous sclerosis complex (TSC), Cowden syndrome (CS) and hereditary paraganglioma/pheochromocytoma (otherwise known as succinate dehydrogenase associated RCC, SDH-RCC).

Hereditary forms of kidney cancer are estimated to account for 3-8\% of newly diagnosed kidney cancers ${ }^{4}$. Identification of patients with a genetic syndrome is important, as it facilitates screening of the affected individual for clinical manifestations associated with the syndrome, as well as detection of affected family members. Furthermore, treatment approaches for patients with a hereditary kidney cancer syndrome differ from those offered to patients with sporadic forms ${ }^{5}$. Examples of this include advocating for aggressive treatment for patients with HLRCC, whereas conservative management is favored for those with VHL. Moreover, patients with a hereditary kidney cancer syndrome benefit from involvement of a multidisciplinary team. Hence, early identification of high-risk patients allows clinicians to alter management with the goal of reducing morbidity and mortality in this unique patient population.

Current guidelines for referral of patients with a suspected hereditary kidney cancer syndrome have been developed by several committees, including the Canadian Urological Association (CUA) and American College of Medical Genetics and Genomics and the National Society of Genetic Counselors (ACGM/NSGC) ${ }^{6,7}$. According to these guidelines, referral to medical genetics is recommended for patients with a diagnosis of RCC and features suggestive of a hereditary syndrome, which are defined as high-risk features in this study, and include: early onset of disease, bilateral or multifocal tumors, family history of RCC, non-clear cell histology with unusual features, and clinical characteristics or associated tumors consistent with a hereditary kidney cancer syndrome. These guidelines assist providers in identifying high-risk patients, however the evidence for these recommendations lacks support from the literature and there remains controversy as to which patients should be referred for counseling.

To date, there is a paucity of data regarding the outcomes of patients identified as being high-risk for hereditary kidney cancer syndromes that have been referred for genetic counseling in a real-life clinical setting. The only published study to our knowledge includes a retrospective cohort of patients assessed by a medical genetics unit at a major cancer center ${ }^{8} .120$ patients with suspected hereditary RCC or Lynch syndrome were referred for genetic counselling, of which 43 underwent testing for a hereditary RCC syndrome. In this cohort, young age predicted presence 
of a hereditary RCC syndrome, with a median age of 48.5 years in those testing positive. Family history, presence of syndromic manifestations, and number of primary malignancies were not associated with a positive test. These results highlight the importance of referring young patients for genetic counseling, however the study is limited by a small sample size and highly selected population.

Based on currently available data, a knowledge gap remains with respect to which patients diagnosed with RCC may benefit from a referral to medical genetics. The primary outcome of this study is to determine risk factors associated with a positive genetic test result in a contemporary real-life cohort of patients referred to a regional medical genetics unit for evaluation of a hereditary RCC syndrome.

\section{Methods}

Using retrospective data, patients referred to Maritime Medical Genetics Service (MMGS, IWK Health Center, Halifax, Nova Scotia, Canada) for evaluation of a hereditary RCC syndrome from January 2006 until December 2017 were identified. Only patients referred for a hereditary RCC syndrome were included in this analysis. Patient referrals from providers were not based on prespecified referral criteria. MMGS defined a distinct set of criteria used for making decisions regarding genetic testing in patients. These include: all patients age $<50$ with RCC; multifocal tumors in patients age $<60$; bilateral tumors in patients age $<70$; personal history of RCC and one relative with RCC (up to third-degree); personal history of RCC with 3 or more first-degree relatives with RCC and one diagnosed at age $<60$.

The criteria for testing patients at MMGS are chosen based on best available evidence as to which patients may carry hereditary RCC mutations. The group meets at a consensus meeting regularly. At regular intervals, this group re-reviews the literature and updates testing criteria; the last changes relating to RCC occurred in 2016. The group also reviews testing criteria from other Canadian institutions to assure that MMGS criteria resembles closely the criteria that is used at other centers nationwide. Notably, the evidence from the literature on this topic to date stems from low-quality evidence, and therefore expert consensus from geneticists and genetic counselors is on occasion required for decisions regarding testing. Hence, any patient referred that does not strictly meet MMGS criteria is discussed at the group consensus meeting (held monthly), comprised of genetic counsellors and medical geneticists specializing in oncology. Decision to proceed with testing is reached by the group and is based on expert opinion, unless there is an update on the topic in the literature. We do not have data available on how many patients in our cohort were discussed at group consensus meetings, however the overall rate of patients discussed at these meetings is less than $5 \%$, and therefore would comprise a small portion of the patient population.

Clinical, pathologic and genetic analysis data was obtained for those patients that were tested for a hereditary RCC syndrome. Clinical data collected includes: age at time of diagnosis, 
sex, date at time of referral, date patient was seen by a genetics counselor, referral source, reason for referral, past medical history, family history, presence of dermatologic findings, multifocal and/or bilateral tumors and presence of recurrence.

Genetic testing was performed by the Clinical Genomic Laboratory, IWK, Halifax; Leeds Genetics Laboratory (Molecular Genetics), St. Jame's University Hospital, Leeds, UK or Blueprint Genetics, Helsinki, Finland. The most commonly used panel for genetic testing at MMGS included FH, FLCN, MET, PTEN, SDHB, SDHC, SDHD, TMEM127 and VHL. Before the availability of panel testing, testing of specific genes, mainly VHL, was left at the discretion of the genetic counselor and geneticist based on their expert review of the case at hand.

\section{Statistical analysis}

Logistic regression analysis was used to determine predictors of a positive test. The outcome variable was the result of the genetic test $(0=$ negative, $1=$ positive $)$. Explanatory variables tested for association with a positive test were age $(<=50,>50)$, pathology (five categories), multifocal and/or bilateral tumors (bilateral or multifocal, non bilateral or multifocal), positive family history of a genetic syndrome or RCC in a first or second degree relative (positive, negative), dermatologic findings (yes, no), and presence of hereditary RCC syndrome-associated tumors outside the kidney (yes, no). Age was chosen as a binary variable over continuous to reflect current guideline criteria. The association of more than two of these variables with a positive result was also tested. Odds ratio (OR), 95\% confidence interval (CI) and p-value were reported (a $\mathrm{p}<0.05$ was considered statistically significant). For significant predictors, sensitivity and specificity were assessed. Univariable analysis (instead of multivariable) was chosen because 6 variables were explored as predictors within a data set in which only 5 cases were positive, resulting in convergence of the solution not being achieved. Statistical analyses were performed using STATA version 15.0 (Stata Corp, College Station, TX).

\section{Results}

Between 2006 and 2017, 135 patients who had a diagnosis of RCC were referred to medical genetics for evaluation of a hereditary kidney cancer syndrome at MMGS. 102 patients were evaluated by a genetics counselor and 75 underwent genetic testing. One patient was excluded because the genetic testing results were not available, resulting in a total of 74 patients that were included in the final analysis. Of the 74 patients, five tested positive for a hereditary RCC syndrome. Three patients tested positive for BHD, one for CS and one for VHL (Figure 1). Four patients tested positive for a variant of unknown significance (VUS) for a hereditary RCC associated gene. The four VUS identified included mutations in the TMEM127, FH, FLCN and TSC2 genes. For the purposes of analysis, these patients were deemed to have a negative genetic test result, as finding of a VUS did not always alter clinical management for the affected individual (ie. through individualized screening or testing of family members). Patients with a 
VUS were screened and monitored based on each individualized clinical scenario and family history.

Baseline characteristics of all patients are summarized in Table 1. The median age at time of referral was 52 years. Clear cell histology was identified in in the majority of patients (53\%). Multifocal or bilateral disease was seen in $42 \%$ of patients, and positive family history of RCC or a genetic syndrome in a first or second degree relative was observed in $44 \%$. Dermatologic findings and presence of associated tumors outside the kidney were found in a minority of patients ( $9 \%$ and $15 \%$, respectively). Associated tumors were defined as any extra-renal tumor known to be associated with a hereditary RCC syndrome.

Characteristics of patients with a positive genetic test are summarized in Table 2. The median age at time of referral was 49 years. Three patients tested positive for the FLCN mutation, one for PTEN and one for VHL. All patients who tested positive for BHD had facial fibrofolliculomas, as did the patient who tested positive for CS. Family history of RCC or a genetic syndrome were present in the patients who tested positive for CS and VHL; BHD patients did not have a positive family history. All patients with a positive genetic test result had more than two high-risk features on history.

The potential high-risk features included: age $<50$, positive family history, multifocal and/or bilateral tumors, presence of associated tumors outside the kidney, dermatologic findings and non-clear cell histology. With the exception of dermatologic findings, these high-risk features independently were not predictive of a positive genetic test result in our patient population (Table 3 ). There was a strong association between a positive genetic test result and dermatologic findings $(\mathrm{OR}=87.99$, CI [7.38, 1048.74], $\mathrm{p}<0.001$, sensitivity $=80 \%$, specificity $=95.7 \%$ ). Multifocality and bilaterality were also tested as separate variables in a secondary analysis, and there was no association with a positive test result. All subjects with a positive genetic test had the presence of more than two high risk features $(100 \%$ sensitivity and specificity).

Individuals who received a positive genetic test result were given information regarding risks to other family members and instructions as to how these family members could proceed with genetic counselling. In most cases, this led to genetic investigations in other family members. Individuals receiving a positive genetic test result also received a review of standard increased screening and/or medical management options based on the condition in question. All individuals with a positive genetic result were recommended to continue with screening already in place or received additional recommendations and/or referrals.

\section{Discussion}

There is a paucity of data regarding outcomes of patients with suspected hereditary RCC referred for genetic testing. Herein, we report that dermatologic findings and presence of more than two high-risk features are the only predictors of a positive genetic test result in a contemporary real- 
life cohort of patients referred for genetic testing of a suspected hereditary kidney cancer syndrome. To our knowledge, our study represents the largest of its kind in the literature to date.

Previous studies evaluating outcomes of patients with kidney cancer referred for testing of a RCC-associated syndrome are limited to a single retrospective study ${ }^{8}$. The authors analyzed 43 patients that underwent testing for a hereditary RCC at a large volume center and found that only age was predictive of a positive test result. Several studies have also suggested that young age is a sign of hereditary $\mathrm{RCC}^{9,10}$. In our patient population, age was not associated with a positive test result, however it is likely that very young patients are at increased risk of harboring a genetic mutation based on larger population based studies.

Other clinical features, such as family history, non-clear cell histology, multifocal/bilateral tumors, as well as presence of other cancers may be suggestive of a hereditary predisposition to $\mathrm{RCC}^{5}$. These features have been determined as risk factors based on classic phenotypic manifestations in patients known to carry a genetic mutation. They have, however, not been validated as predictors of a positive test result in patients presenting with RCC not yet diagnosed with a hereditary syndrome. Our analysis demonstrates that in a real-life setting, most features with a known association to hereditary RCC in fact do not predict a positive genetic test result. This suggests that classic phenotypic hereditary RCC manifestations are also prevalent in the general population and their presence does not necessarily predict the presence of a genetic syndrome. This carries important implications for providers selecting patients for referral for genetic testing.

In addition to known hereditary RCC genetic mutations, rare cancer associated genes that have not classically been associated with RCC syndromes are emerging. A recent study identified 254 patients with advanced RCC and found that most individuals from this cohort carried germline mutations in non-RCC associated genes ${ }^{11}$. Patients with non-clear cell histology were more likely to harbor a mutation. Interestingly, although many patients met guideline criteria for genetic testing, a large proportion (36\%) of patients with detected RCC associated mutations would have been missed based on referral criteria alone. This highlights the limitations of current guidelines and supports the findings of our study. The clinical indication for testing patients with RCC for novel and rare germline mutations remains limited, and therefore providers must optimize the identification of appropriate patients for genetic evaluation until our scientific knowledge on this subject expands.

The identification and subsequent referral of high-risk patients remains a challenge for clinicians. A Surveillance, Epidemiology, and End Results Program (SEER) database study identified all patients with a known kidney cancer diagnosis, and found that $24 \%$ of patients met ACMG/NSGC genetic referral criteria based on RCC histologic subtypes alone, suggesting that patients are under-referred for genetic counseling ${ }^{12}$. Provider knowledge, accessibility to a genetics unit, possible lack of clinical impact and wait times have been identified as barriers to 
genetic testing referrals for patients with suspected hereditary RCC amongst Canadian urologists $^{13}$. From a patient perspective, it has been shown that cost of testing in women with breast cancer is an established barrier for undergoing genetic assessment, and therefore patients often decline referral to a geneticist ${ }^{14}$. It is plausible that improvement and validation of current referral criteria with studies such as ours will guide clinicians toward counseling appropriate patients for genetic testing.

Our data suggests that most patients with high-risk features as outlined by current guideline criteria will not test positive for an RCC associated syndrome. We demonstrate for the first time that the presence of more than two high-risk features is perfectly correlated with a positive test result in patients diagnosed with RCC who are referred for evaluation of a hereditary kidney cancer syndrome. This subset of patients is most likely to benefit from a referral to medical genetics, and providers should be aware of this, as it is not currently reflected in guidelines. We hypothesize that the presence of high-risk features in isolation is prevalent in sporadic RCC, but that patients with hereditary RCC are more likely to present with several risk factors, however this needs further validation.

We report that dermatologic findings, specifically facial fibrofolliculomas, are highly correlated with a positive genetic test result (OR (87.99)). Dermatologic skin lesions are prevalent in several hereditary RCC syndromes, including BHD (fibrofolliculomas), TSC (angiofibromas, shagreen patches) and HLRCC (cutaneous leiomyomata) ${ }^{15}$. Of interest, dermatologic findings are not listed as specific referral criteria within the ACGM/NSGC guidelines, despite their known association with hereditary RCC syndromes. In this study, all positive dermatologic findings were fibrofolliculomas and therefore we are unable to draw conclusions regarding the association of other lesions with a positive test result. Furthermore, the association of dermatologic lesions with a positive test result may be a reflection of a high proportion of patients testing positive for BHD, while syndromes not associated with classic skin findings (such as VHL) were not as common in this patient cohort. Nonetheless, all patients testing positive for a genetic syndrome were referred to medical genetics by a urologist and we therefore emphasize the importance of a dermatologic evaluation in any patient diagnosed with RCC with appropriate referrals to dermatology for suspicious lesions.

Our study has some limitations. In this cohort, only $7 \%$ tested positive for a hereditary kidney cancer syndrome. This contrasts with findings in other studies, where a higher proportion of patients tested positive $(30 \%)^{8}$. Our panel did not routinely test for other genes associated with RCC, such as BAP1, TP53, TSC1 and TSC2, or genes less typically associated with RCC, like BRCA1 and BRCA2, and this may partly account for the difference in detection rates. Given the rarity of these mutations in the population, and the low association of TSC1/2 with RCC, it is unlikely that this would have altered our findings, but could be impactful. Not all patients referred for genetics evaluation underwent a complete assessment. Up to $50 \%$ of patients referred 
for genetic testing were not tested due to patient refusal, loss of follow up or discordant indications for testing. It is possible that patients with a positive test result may have been included in this cohort.

The majority of patients in this study were tested based on referral criteria developed by the genetics group at our center, similar to current guidelines, and using a standardized hereditary RCC genetics panel. With the advent of new technologies and improvements in the capacity and accessibility of genetic testing, panels and testing options have required alteration over time. As a result, a panel would not always have been available, and patients identified earlier in the cohort would have had more limited testing. In these cases, testing of specific genes was determined by the genetic counselor and geneticist based on clinical data, expert opinion, and best available evidence at that time. As our knowledge of germline mutations continues to expand, it is expected that panel testing will continue to change over time to reflect currently available literature. This type of heterogeneity is resolvable only in the setting of a prospective trial design. An ideal prospective trial would test all patients with a diagnosis of RCC for germline mutations using a variety of known cancer genes, not limited to RCC-specific mutations (as was performed in the study by Caro et al. ${ }^{11}$ ). This would facilitate the identification of high-risk clinical features in an unbiased cohort that was not selected for referral, with the understanding that the field of germline mutations research is rapidly evolving and testing panels require continued updating. Nonetheless, our findings represent a real-life setting and are therefore clinically relevant to a diverse patient population within various clinical scenarios. Other limitations of this study include its retrospective, single-center nature and small cohort, which will require corroboration in a larger multi-institutional setting.

\section{Conclusions}

In the largest study of its kind to date, we have identified dermatologic findings and the presence of more than two high-risk features as the only predictors of a positive genetic test result in patients with a suspected hereditary RCC syndrome. Current guidelines do not reflect the importance of our findings, and we hypothesize that implementation of genetic referrals based on these criteria will improve identification of patients with hereditary RCC syndromes. Limitations of this study include a small retrospective and single center cohort. These findings require further validation in multi-institutional setting. 


\section{References}

1. Canadian Cancer Statistics Advisory Committee. (2019). Canadian Cancer Statistics 2019. Toronto, ON: Canadian Cancer Society. https://www.cancer.ca/en/cancerinformation/cancer-101/canadian-cancer-statistics-publication/. Accessed September 16, 2019.

2. Moch H, Cubilla AL, Humphrey PA et al. The 2016 WHO Classification of tumours of the urinary system and male genital organs - Part A: Renal, penile, and testicular tumours. Eur Urol 2016;70:93-105.

3. Maher, E. Hereditary renal cell carcinoma syndromes: diagnosis, surveillance and management. World J Urol 2018;36:1891-98.

4. Linehan WM, Srinivasan R, Schmidt LS. The genetic basis of kidney cancer: A metabolic disease. Nat Rev Urol 2010;7:277-85.

5. Shuch B and Zhang J. Genetic predisposition to renal cell carcinoma: Implications for counseling, testing, screening, and management. J Clin Oncol 2018. Epub 2018 Oct 29.

6. Hampel H, Bennett RL, Buchanan A et al. A practice guideline from the American College of Medical Genetics and Genomics and the National Society of Genetic Counselors: Referral indications for cancer predisposition assessment. Genet Med 2015;17:70-87.

7. Reaume NM, Graham GE, Tomiak E et al. Canadian guideline on genetic screening for hereditary renal cell cancers. Can Urol Assoc J 2013;7:319-23.

8. Stratton KL, Alanee S, Glogowski EA et al. Outcome of genetic evaluation of kidney cancer patients referred for suspected hereditary cancer syndromes. Urol Oncol 2016;34: 238.e1-e7.

9. Schuch B, Vourganti S, Ricketts CJ et al. Defining early onset kidney cancer: implications for germline and somatic mutation testing and clinical management. J Clin Oncol 2014;32:431-37.

10. Nguyen KA, Syed JS, Espenschied CR et al. Advances in the diagnosis of hereditary kidney cancer: Initial results of a multigene panel test. Cancer 2017;123:4363-71.

11. Carlo MI, Mukherjee S, Mandelker D et al. Prevalence of germline mutations in cancer susceptibility genes in patients with advanced renal cell carcinoma. JAMA Oncol 2018;4:1228-35.

12. Truong H, Hegarty SE, Gomella LG et al. Prevalence and characteristics of patients with suspected inherited renal cell cancer: Application of the ACMG/NSGC genetic referral guidelines to patient cohorts. J Genet Couns 2017;26:548-55.

13. Violette PD, Kamel-Reid S, Graham GE et al. Knowledge of genetic testing for hereditary kidney cancer in Canada is lacking: The results of the Canadian national hereditary kidney cancer needs assessment survey. Can Urol Assoc J 2014;8:E832-E840.

14. Weldon CB, Trosman JR, Gradishar WJ, et al. Barriers to the use of personalized medicine in breast cancer. J Oncol Pract 2012;8:E24-E31.

15. Gaur S, Turkbey B, Choyke P. Hereditary renal tumor syndromes: update on diagnosis and management. Semin Ultrasound CT MR 2017;38:59-71. 


\section{Figures and Tables}

Fig. 1. Patient evaluation. BHD: Birt-Hogg-Dube syndrome; RCC: renal cell carcinoma; TSC: tuberous sclerosis complex; VHL: Von Hippel-Lindau.

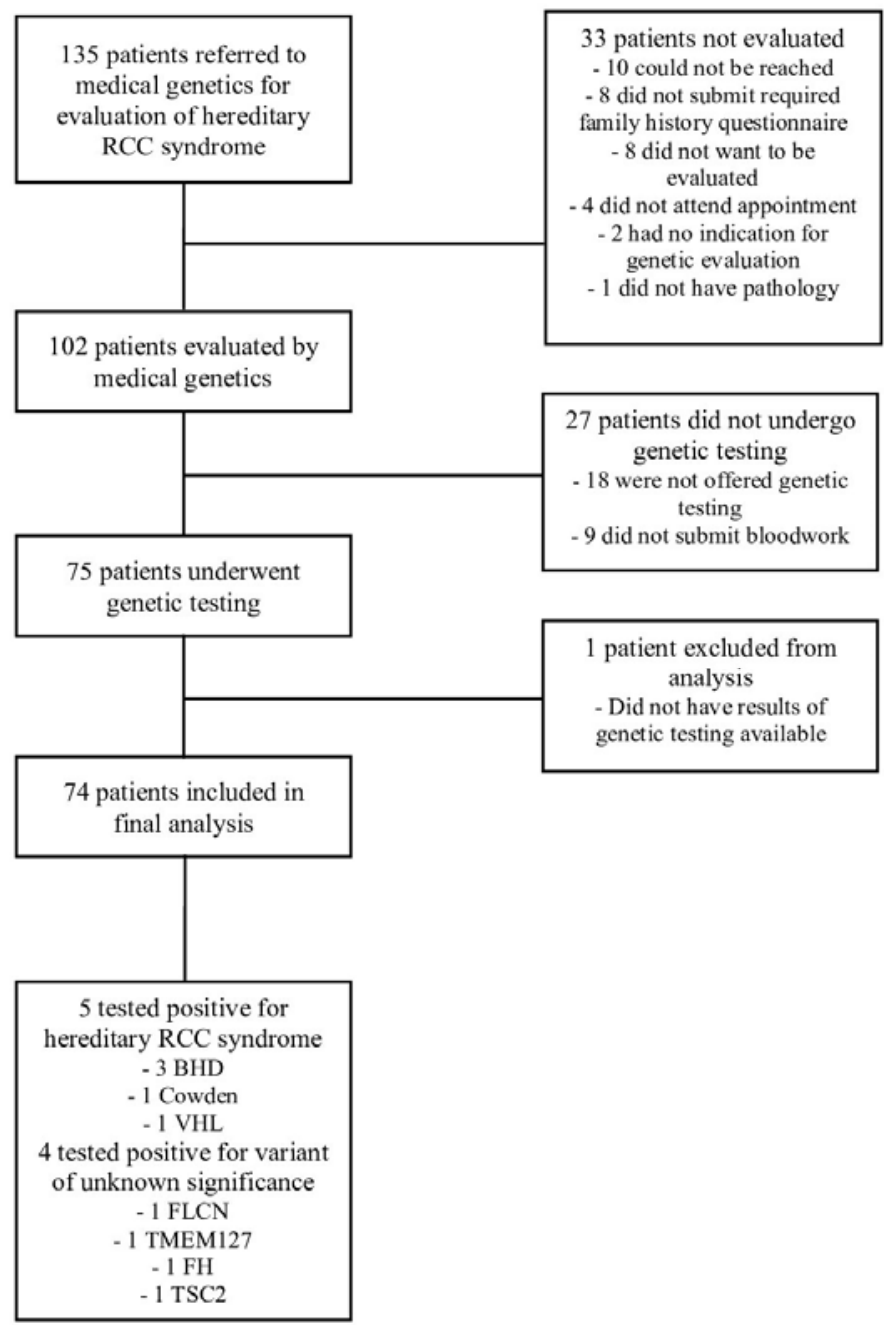




\begin{tabular}{|c|c|c|c|}
\hline \multirow[b]{2}{*}{ Characteristic } & \multicolumn{3}{|c|}{ n (\%) } \\
\hline & $\begin{array}{c}\text { All patients } \\
(\mathrm{n}=74)\end{array}$ & $\begin{array}{l}\text { Negative test } \\
\text { result }(\mathrm{n}=69)\end{array}$ & $\begin{array}{l}\text { Positive test } \\
\text { result }(n=5)\end{array}$ \\
\hline \multicolumn{4}{|l|}{ Sex } \\
\hline Male & $39(53 \%)$ & $38(55 \%)$ & $1(20 \%)$ \\
\hline Female & $35(47 \%)$ & $31(45 \%)$ & $4(80 \%)$ \\
\hline \multicolumn{4}{|l|}{ Age } \\
\hline$\leq 50$ & $35(47 \%)$ & $32(46 \%)$ & $3(60 \%)$ \\
\hline$>50$ & $39(53 \%)$ & $37(54 \%)$ & $2(40 \%)$ \\
\hline Median age at time of referral & 52 & 52 & 49 \\
\hline \multicolumn{4}{|l|}{ Pathology } \\
\hline Clear-cell RCC & $39(53 \%)$ & $38(55 \%)$ & $1(20 \%)$ \\
\hline Papillary RCC & $17(23 \%)$ & $15(22 \%)$ & $2(40 \%)$ \\
\hline $\begin{array}{l}\text { Oncocytic/hybrid neoplasm or chromophobe } \\
\text { RCC }\end{array}$ & $11(15 \%)$ & $9(13 \%)$ & $2(40 \%)$ \\
\hline AML & $1(1 \%)$ & $1(1 \%)$ & 0 \\
\hline$\varnothing$ Other & $3(4 \%)$ & $3(4 \%)$ & 0 \\
\hline$¥$ Unknown & $3(4 \%)$ & $3(4 \%)$ & 0 \\
\hline Multifocal or bilateral tumors & $31(42 \%)$ & $28(41 \%)$ & $3(60 \%)$ \\
\hline Positive family history & $31(42 \%)$ & $29(42 \%)$ & $2(40 \%)$ \\
\hline Presence of dermatological findings & $7(9 \%)$ & $3(4 \%)$ & $4(80 \%)$ \\
\hline Presence of tumors outside the kidney & $11(15 \%)$ & $9(13 \%)$ & $2(40 \%)$ \\
\hline Presence of more than two high-risk features & $17(23 \%)$ & $12(17 \%)$ & $5(100 \%)$ \\
\hline TNM stage (pathological) & & & \\
\hline pT1NanyM0 & $41(55 \%)$ & $39(57 \%)$ & $2(40 \%)$ \\
\hline pT2NanyM0 & $8(11 \%)$ & $8(12 \%)$ & 0 \\
\hline pT3N $\mathrm{N}_{\text {any }} \mathrm{M} 0$ & $10(14 \%)$ & $9(13 \%)$ & $1(20 \%)$ \\
\hline pT4NanyM0 & $1(1 \%)$ & $1(1 \%)$ & 0 \\
\hline pT unknown & $14(19 \%)$ & $12(17 \%)$ & $2(40 \%)$ \\
\hline $\mathrm{pT}_{\text {any }} \mathrm{N}_{\text {any }} \mathrm{M} 1$ (at presentation) & $5(7 \%)$ & $5(7 \%)$ & 0 \\
\hline
\end{tabular}

$\varnothing 1$ tubulopapillary; 1 RCC associated with neuroblastoma; 1 unclassified; $¥ 1$ pathology report unavailable; 1 on active surveillance without biopsy; 1 pathology report did not specify RCC subtype. AML: angiomyolipoma; RCC: renal cell carcinoma. 


\begin{tabular}{|c|c|c|c|c|c|c|c|c|c|c|}
\hline Sex & Age & Mutation & Syndrome & Pathology & $\begin{array}{l}\text { Associated } \\
\text { tumors }\end{array}$ & $\begin{array}{l}\text { Dermatological } \\
\text { findings }\end{array}$ & $\begin{array}{l}\text { Family } \\
\text { history }\end{array}$ & Bilateral & Multifocal & $\begin{array}{c}\text { Referral } \\
\text { source }\end{array}$ \\
\hline $\mathrm{F}$ & 36 & PTEN & Cowden & $\begin{array}{c}\text { RCC, mixed } \\
\text { papillary type } 2 \\
\text { with component } \\
\text { of clear cell }\end{array}$ & $\begin{array}{c}\text { Pelvic/liver } \\
\text { hemangioma, } \\
\text { shoulder } \\
\text { angiolipoma, } \\
\text { thyroid } \\
\text { neoplasm with } \\
\text { high-risk } \\
\text { features; Chiari } \\
\text { type 1 } \\
\text { malformation, } \\
\text { vascular tumor } \\
\text { left eyelid, } \\
\text { multiple AVM }\end{array}$ & $\begin{array}{c}\text { Facial } \\
\text { fibrofolliculomas; } \\
\text { chest } \\
\text { telangiectasia; } \\
\text { forehead angioma }\end{array}$ & $\begin{array}{l}\text { Father - } \\
\text { RCC and } \\
\text { Cowden }\end{array}$ & No & No & Urologist \\
\hline $\mathrm{F}$ & 76 & FLCN & BHD & $\begin{array}{c}\text { (a) RCC, } \\
\text { papillary type } 2 \\
\text { (b) RCC, } \\
\text { oncocytic } \\
\text { papillary type } 2\end{array}$ & None & $\begin{array}{c}\text { Facial } \\
\text { fibrofolliculom, } \\
\text { trichodiscomas }\end{array}$ & None & Yes & No & Urologist \\
\hline $\mathrm{F}$ & 47 & FLCN & BHD & $\begin{array}{l}\text { Oncocytic } \\
\text { neoplasm, favor } \\
\text { hybrid oncocytic } \\
\text { neoplasm }\end{array}$ & None & $\begin{array}{c}\text { Facial } \\
\text { fibrofolliculomas }\end{array}$ & None & No & No & Urologist \\
\hline
\end{tabular}




\begin{tabular}{|c|c|c|c|c|c|c|c|c|c|c|}
\hline $\mathrm{F}$ & 56 & FLCN & BHD & $\begin{array}{l}\text { (a) } 6 \text { oncocytic } \\
\text { renal cell } \\
\text { neoplasms } \\
\text { consistent with } \\
\text { multiple hybrid, } \\
\text { oncocytic, } \\
\text { chromophobe } \\
\text { tumours } \\
\text { (b) } 3 \text { mixed } \\
\text { oncocytoma and } \\
\text { chromophobe } \\
\text { tumours }\end{array}$ & None & $\begin{array}{c}\text { Facial } \\
\text { fibrofolliculomas }\end{array}$ & None & Yes & Yes & Urologist \\
\hline $\mathrm{M}$ & 49 & VHL & VHL & RCC, clear-cell & $\begin{array}{c}\text { Spinal } \\
\text { hemangioblasto } \\
\text { mas, pancreatic } \\
\text { cyst }\end{array}$ & None & $\begin{array}{c}\text { Father - } \\
\text { kidney } \\
\text { cancer } \\
\text { and renal } \\
\text { cyst }\end{array}$ & Yes & Yes & Urologist \\
\hline
\end{tabular}

AVM: angiomyolipoma; BHD: Birt-Hogg-Dube syndrome; F: female; M: male; RCC: renal cell carcinoma; VHL: Von Hippel-Lindau. 


\begin{tabular}{|l|c|c|c|c|}
\hline \multicolumn{5}{|l|}{ Table 3. Clinical predictors for a positive genetic test result (univariate analysis) } \\
\hline Variable & OR & LCI & UCI & p \\
\hline Age (<50 vs. >50) & 1.73 & 0.27 & 11.03 & 0.56 \\
Pathology & & & & \\
$\quad$ Papillary & 5.07 & 0.43 & 60.14 & 0.20 \\
$\quad$ Oncocytic/hybrid or chromophobe & 2.91 & 0.83 & 10.18 & 0.096 \\
Multifocal or bilateral tumors & 2.20 & 0.34 & 14.0 & 0.41 \\
Positive family history & 0.92 & 0.14 & 5.86 & 0.093 \\
Dermatological findings & 88.0 & 7.38 & 1048.74 & 0.00 \\
Tumors outside the kidney & 4.44 & 0.65 & 30.36 & 0.13 \\
More than two high-risk features & $* *$ & $* *$ & $* *$ & $* *$ \\
\hline
\end{tabular}

*Clear-cell, AML, other and unknown histology were tested but OR could not determined (not enough sample size). ${ }^{* *}$ Presence of more than two high risk features predicts probability of genetic test result perfectly and therefore odds ratio can not be determined. LCI: lower confidence interval (95\%); OR: odds ratio; UCI: upper confidence interval (95\%). 